\title{
Exploiting the Misalignment of the Serrated Trailing Edges for Improved Aerofoil Broadband Noise Reduction
}

\author{
P.C. Woodhead ${ }^{1}$, T.P. Chong ${ }^{2}$, J.G. Wissink ${ }^{3}$
}

Brunel University London, Uxbridge, UB8 3PH, UK

\begin{abstract}
This paper presents the experimental results of adding variable flap angles to the serrated trailing edges and their effects on the self-noise radiation of an aerofoil. The investigation included aeroacoustics and wake flow measurements on an NACA 65-(12)10 aerofoil. This paper explores further combinations of positive and negative serration flap angles, $\phi_{U}$ and $\phi_{L}$ respectively, across the spanwise extent of the trailing edge. A new parameter, the serration oscillation wavelength $\left(\lambda^{\prime}\right)$, was also included. The study investigates five individual cases, Baseline (B), Straight-Serration (SS), sIngle-Flapped-Serration (IFS), Multi-FlappedSerration (MFS), Split-Flapped-Serration (SFS) and Spanwise-Wavy-Serration (SWS). The serration amplitude and wavelength remained consistent as $30 \mathrm{~mm}$ and $3.3 \mathrm{~mm}$ respectively. It is clear that all the "misaligned" trailing edges produce different noise characteristics compared to the conventional SS trailing edge. At low-to-mid frequency range, the conventional SS trailing edge still outperforms both the MFS and SFS trailing edges. However, from mid-to-high frequency, both the MFS and SFS trailing edges can achieve higher noise reduction than the SS trailing edge considerably. At very high frequency, where the SS trailing edge would otherwise experience noise increase, there is no noise increase for both the MFS and SFS trailing edges because the periodic misalignment reduces the crossflow component in the space between adjacent members of the serration. The SWS trailing edge produces the same level of noise reduction as the SS trailing edge across the low-to-mid frequency range. From mid-to-high frequency range, the SWS trailing edge outperforms the SS trailing edge, but the level of further noise reduction it can achieve is less than those of MFS and SFS trailing edges. At very high frequency, the SWS begins to experience slightly noise increase over the baseline, $B$, trailing edge. However, the increase in noise level by the SWS trailing edge at this high frequency range is still less than that by the SS trailing edge. The near-wake flow measurement results provide some explanations for the mechanisms underpinning the broadband noise reduction and high frequency noise suppression by these trailing edge devices.
\end{abstract}

\section{Introduction}

O ver the next 15 years, the European Union (EU) will make significant changes to the wind turbine sector. These changes will take into consideration the recent development in the regulations for wind energy [1]. Future scenarios have been drawn up to year 2030 to increase the wind energy capacity in the EU. One of the main targets

${ }^{1} \mathrm{PhD}$ student, Department of Mechanical, Aerospace and Civil Engineering, philip.woodhead@brunel.ac.uk. (corresponding author)

${ }^{2}$ Senior lecturer, Department of Mechanical, Aerospace and Civil Engineering, t.p.chong@brunel.ac.uk. Member AIAA member

${ }^{3}$ Senior lecturer, Department of Mechanical, Aerospace and Civil Engineering, jan.wissink@brunel.ac.uk. NonAIAA member 
is to produce 320 Giga-watts (GW) of wind energy per annum in year 2030, where $79 \%$ of which will be generated by the onshore wind turbines, and the remaining from the offshore wind turbines. This ambitious target highlights the major drive to invest heavily on the onshore wind farms in the EU member states. However, a major concern in achieving this energy target is the inevitably the increase of aerodynamic noise pollution from the wind turbine blades. To protect residents, maximum noise levels are set that may not be exceeded. To stay within the noise limit, wind turbines often need to operate at reduced speed, which causes the wind energy effectively more expensive to generate. Reduction of blade noise without reducing the rotor speed would therefore make wind energy cheaper. Therefore wind turbine manufacturers are actively seeking new technology to further reduce the level of the selfnoise of their turbine blades. Wind turbine airfoil noise can be generated at the leading edges of the blades, through interaction with the atmospheric turbulence, or at the trailing edges, where turbulence in the boundary layer that develops on the blade surface scatters into sound [2]. This paper only focuses on the trailing edge self-noise.

The bio-inspired passive flow control represents an attractive method to achieve aerodynamic noise reduction. In particular the use of serration is shown to be one of the most effective passive devices to reduce the trailing edge noise of aerofoils. The serration draws inspiration from the unique features found in an owl wing. Studies have shown that when the serration is mimicked and applied to the trailing edge of an aerofoil, not only it could lead to reduction in the aerodynamic drag [3,4], but also the reduction on the self-noise radiation [2, 5-9, 20]. The serration can also be formed in several configurations, such as the M-shaped [4, 11], wavy [10] and sawtooth [2, 5, 8, 9, 12]. The focus of this study is on the sawtooth serration.

Amiet [13] explained the connection between the far-field acoustic pressure and the near-field surface pressure near the trailing edge. For example, the fully-developed turbulent boundary layer would result in the radiation of broadband noise from the trailing edge when the turbulent energy was scattered from the boundary layer. Amiet [13] also stated a relationship between the power spectral density of the far-field noise $S_{p p}$ and the spanwise correlation length $I_{y}$, the surface pressure spectrum $S_{q q}$ near the trailing edge, and the radiation term $L(\omega)$, in the form of $S_{p p}(\omega)$ $\propto L(\omega) I_{y}(\omega) S_{q q}(\omega)$. Note that $\omega$ is the angular frequency. Reduction in any of these variables $\left(I_{y}, S_{q q}\right.$ and/or $\left.L(\omega)\right)$, which in principle is achievable by introducing serrated sawtooth to the trailing edge, can result in a reduction of the radiated broadband noise.

Howe [20] provides a theoretical expression of the noise reduction by the serrated sawtooth trailing edge at low Mach number flow. The theoretical model stipulates that the serrated inclination angle must be less than $45^{\circ}$ to the freestream direction to be effective in the noise reduction at frequencies $\omega h / U \gg 1$, where $\mathrm{h}$ is the half-length of the sawtooth and $U_{\infty}$ is the freestream velocity. It was also stated that the dimension of the serration must the same order as the turbulent boundary layer thickness $\delta$ near the trailing edge. The studies by Gruber et al. [14] [2013], Moreau and Doolan [7] and Gruber et al. [8] compared their measured noise reduction level of serrated trailing edges to those of predicted by the Howe's model. It was found that the predicted values overestimated the experimental data. Recently, Lyu et al. [15] developed a theoretical model for prediction of sound radiated by serrated trailing edges. The new model provides a better prediction of noise generated by serrated trailing edges, which is underpinned by the mechanism of the destructive interference on the scattered pressure field.

Experimentally, Pröbsting [16] investigated the NACA 0012 aerofoil with interchangeable non-flat plate trailing edges to compare the straight and serrated sawtooth cases. The aim was to examine the volumetric flow field by the tomographic particle image velocimetry (PIV) at the trailing edge. A subset of coherent structures was observed near the trailing edge. Due to the presence of blunt root at the sawtooth, this led to a shedding of longitudinal vortices across the sawtooth surface. Large coherent structures, such as the hairpins or horseshoes, were also observed close to the trailing edge or in between the serrations. The shedding of smaller vortices from the trailing edge was emanated from the space between the serrations. Pröbsting [16] suggested that the broadband noise reduction is likely to be caused by the interaction between the blunt-induced vortex shedding and the hydrodynamic field on the sawtooth surface, which ultimately cause an energy redistribution within the turbulence spectrum of the boundary layer.

The reduction of trailing edge noise by different kinds of sawtooth serrations has been successfully demonstrated in many wind tunnel experiments of Vathylakis et al. [12], León et al. [17] and Chong and Vathylakis [6] and Gruber et al. [8]. Reduction of the trailing edge noise by serration on a real size wind turbine blade has also been shown by Orelemans et al. [2]. Overall, in terms of the level of trailing edge noise reduction, there is a discrepancy 
of the reported values between the wind tunnel experiments (up to $8 \mathrm{~dB}$ ) and the wind turbine blades (about $3 \mathrm{~dB}$ ). Jones and Sandberg [18] observed that the serrated trailing edges had little effect on the directivity of the self-noise radiation. However, many studies have now observed an increase in high frequency noise when a serrated trailing edge is applied. Studies by Oerlemans et al. [2] and Dassen et al. [19] proposed that misalignment of the serrated trailing edge is the reason for the increase of high frequency noise, as the serrations were misaligned with the flow in the wake of the aerofoil. Gruber et al. [8] suggested that the large increase in high frequency noise is a result of cross-flow between the serrations.

Most recently, León et al. [17] and Vathylakis et al. [12] artificially bent their sawtooth serration to facilitate a controlled "flap angle". León et al. [17] investigated the flow and noise characteristics of a NACA 0018 with sawtooth trailing edge. They reported that negative flap angles (flap-down) could degrade the level of broadband noise reduction in comparison to the straight serrations, or even produce higher noise level than the baseline, straight trailing edge case. Such noise increase is usually accompanied by the presence of high turbulence intensity in the wake. They also utilised the stereoscopic PIV to study the serration flap angle and aerofoil incidence to observe the effect of the secondary flow established on the suction and pressure sides of the serrated trailing edge. The results showed the additional formation of streamwise vortex pairs in the cross-flow plane. They observed a strong relationship between the vortices and serration with flap angles rather than the aerofoil incidence. At zero aerofoil incidence and serration flap angle the mean flow behavior near the serration adhered closely to Howe's assumptions of the reduced acoustic scattering efficiency at the presence of oblique edges. Observation of streamlines at the trailing edge tended to align with the edge of the serration on the suction side. In contrast, the opposite occurred to the pressure side with the streamlines tending to become normal at the edge of the serrations.

The most common geometrical variables describing a serration are traditionally the serration amplitude and wavelength. Whilst keeping these variables constant, Vathylakis et al. [12] investigated the sensitivity of the aerofoil broadband noise reduction to a third geometrical variable - serration flap angle, which could be regarded as a form of serration misalignment. Several flap angles were investigated: $\pm 5^{\circ}, \pm 10^{\circ}, \pm 15^{\circ}$, where the flap angle at $0^{\circ}$ is treated as the reference. They found that certain serration flap angles can actually further improve the level of broadband noise reduction. In particular, a flap-up position was generally found to achieve higher level of broadband noise reduction than the flap-down positions. The most optimum flap-up angle is at $+5^{\circ}$, where it outperforms the non-flap angle case from mid-to-high frequencies. However, the $-5^{\circ}$ flap-down angle represents the worst performer amongst all the non-zero flap angle cases because of the consistent noise increase throughout the whole range of frequency. Nevertheless, the largest flap-down angle at $-15^{\circ}$ produces almost the identical level of broadband noise reduction as the non-flap angle case.

The literature has shown significant benefits of trailing edge serrations from the aeroacoustics perspective. However, most studies also observed a consistent increase in the high frequency noise levels. Several studies have suggested the possible reasons for the increase in high frequency noise, which are related to the cross-flow within the trough of the serrations [8], or misalignment of the serrations [2,19]. However, the results from Vathylakis et al. [12] suggest that the concept of misalignment of the serration at the trailing edge could be carefully exploited to further improve the level of the broadband noise reduction. This paper will explore the effects of the periodic and out-of-phase serration misalignment. In particular, three new combinations of the serration geometries: (1) MultiFlapped-Serration, MFS, (2) Split-Flapped-Serration SFS and (3) Spanwise-Wavy-Serration, SWS, will be investigated. 


\section{Experimental setup}

The following sub-sections describe the experimental setup and analysis techniques used within this research, which are similar to those of previous study by Vathylakis et al. [12].
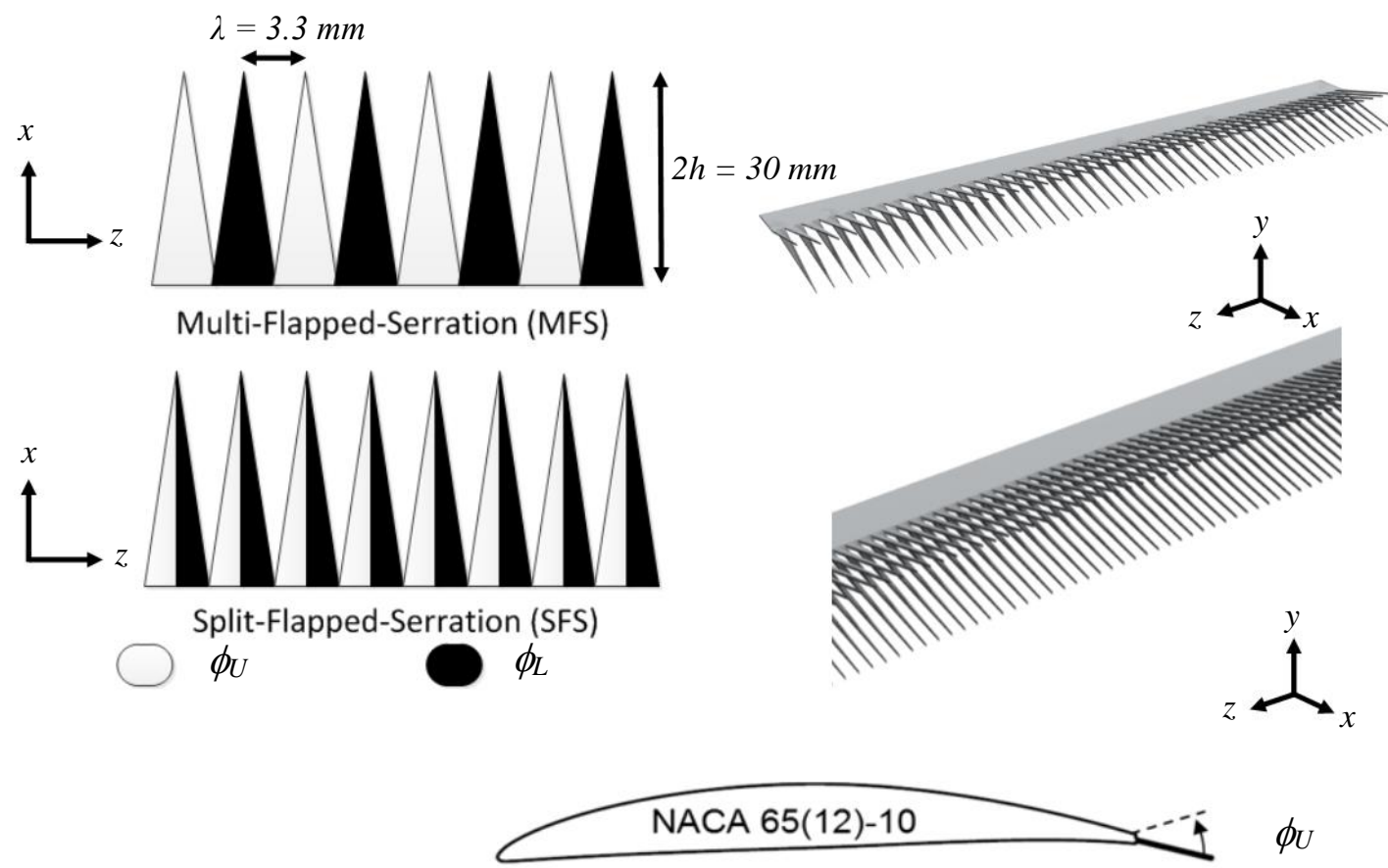

sIngle-Flapped-Serration (IFS)
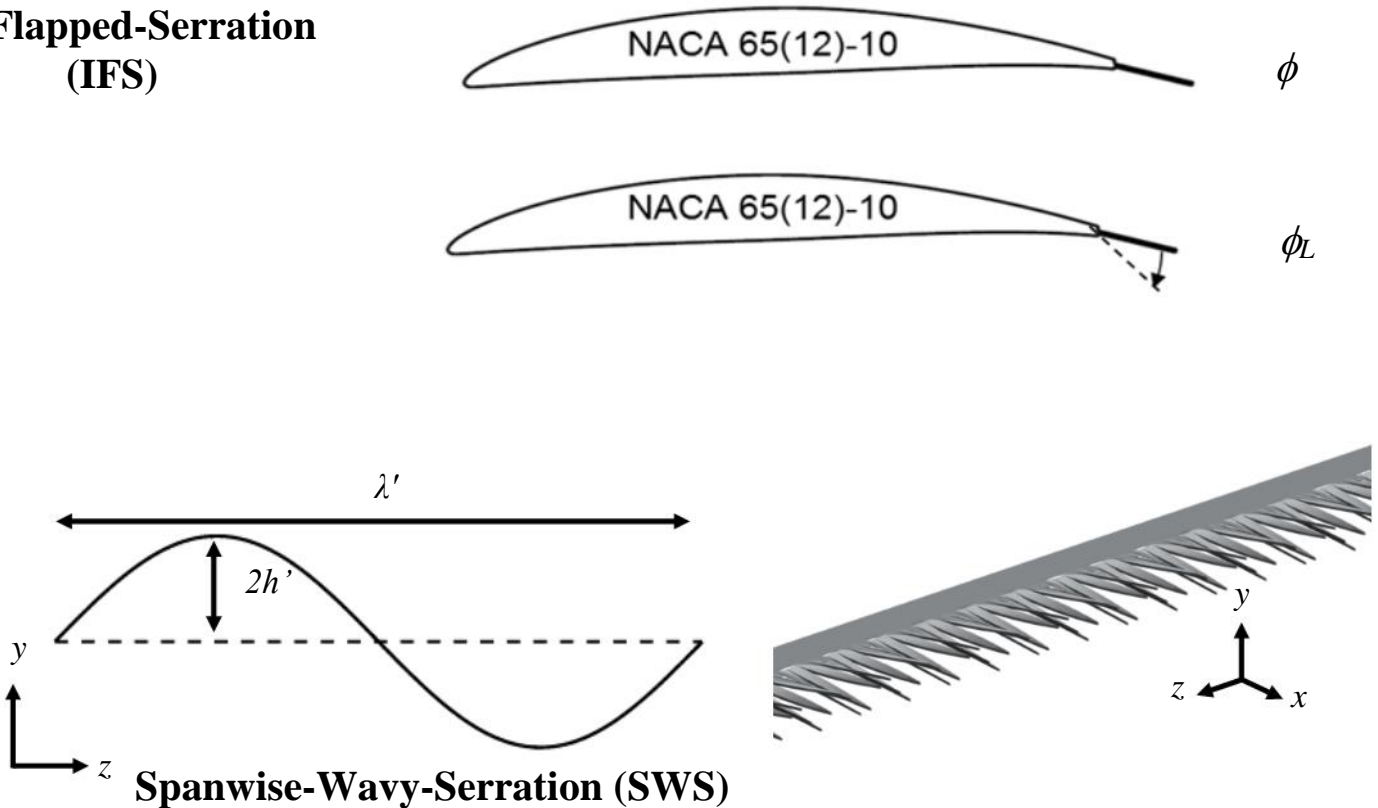

Fig 1 Geometric parameters of the flat plate trailing edge add-on for the NACA65-(12)10: serration amplitude $(2 h)$, serration wavelength $(\lambda)$, flap angles $(\phi)$ and oscillation serration wavelength $(\lambda$ ').

4

American Institute of Aeronautics and Astronautics 


\section{A. Design of the NACA65-(12)10 cambered aerofoil with Flapped Serrated Trailing Edge}

The experiment was conducted on a NACA65-(12)10 cambered aerofoil. This is the same aerofoil used in the previous studies at the Institute of Sound and Vibration Research (ISVR), University of Southampton [8] and most recently at Brunel University London [12]. The NACA65-(12)10 aerofoil had a chord length of $0.16 \mathrm{~m}$ and $0.175 \mathrm{~m}$ for baseline and serration cases respectively and a span-wise length of $0.45 \mathrm{~m}$. The difference in chord length was to achieve the same wetted surface area for the baseline and serrated cases. The NACA 65-(12)10 aerofoil consists of two parts: main body and detachable trailing edges. The main body was manufactured from solid aluminum alloy with pressure taps across the upper and lower surfaces of the aerofoil with a $0.8 \mathrm{~mm}$ slot along the trailing edge. The detachable trailing edges were 3D-printed with a printing resolution of 100 microns $(0.01 \mathrm{~mm})$ for all the cases.

Figure 1 displays the geometric parameters of the trailing edge flat plate cases. These are defined as the serration amplitude $(2 h)$, serration wavelength $(\lambda)$, flap angle $(\phi)$, serration spanwise oscillation amplitude $\left(2 h^{\prime}\right)$ and serration spanwise oscillation wavelength $\left(\lambda^{\prime}\right)$. In a previous study by Vathylakis et al. [12], the serration amplitude and serration wavelength were kept at $2 h=30 \mathrm{~mm}$ and $\lambda=3.3 \mathrm{~mm}$, respectively. The un-serrated, baseline flat plate trailing edge was half the serration amplitude (i.e. $2 \mathrm{~h}=15 \mathrm{~mm}$ ), which would produce the same wetted surface area for both serrated and baseline cases. The current research studies five serration cases: Baseline (B), StraightSerration (SS), sIngle-Flapped-Serration (IFS), Multi-Flapped-Serration (MFS), Split-Flapped-Serration (SFS) and Spanwise-Wavy-Serration (SWS). These acronyms are used throughout this study, and the readers can refer to Fig. 1 for the schematics illustrating these serration cases. The coordinate system adopted in relation to these serration cases is defined as follows: streamwise $(x)$, vertical $(y)$ and spanwise $(z)$.

In this work the main aerofoil body had a $0.8 \mathrm{~mm}$ slot along the rear end, which allows the insertion of the flat plate trailing edges of about $0.8 \mathrm{~mm}$ thickness. The non-zero flap angle trailing edge cases were manufactured with the desired flap angle of $\phi= \pm 5^{\circ}, \pm 10^{\circ}$ and $\pm 15^{\circ}$, and $\lambda^{\prime}=15 \mathrm{~mm}, 30 \mathrm{~mm}$ and $45 \mathrm{~mm}$ and $2 h^{\prime}=2.6247 \mathrm{~mm}, 5.2898 \mathrm{~mm}$ and $8.0385 \mathrm{~mm}$. Note that $\phi_{U}$ and $\phi_{L}$ denote the flap-up and flap-down angles, respectively. Coarse sandpaper strips were applied to both the upper and lower surfaces at $x / \mathrm{C}=0.2$ of the aerofoil to trip the boundary layer into turbulent. Note that $\mathrm{C}$ is the aerofoil chord. The coarse sandpaper had a thickness of $0.95 \mathrm{~mm}$ and width of $10 \mathrm{~mm}$.

\section{B. Wind tunnel facilities and instrumentation}

This section describes the wind tunnel facilities and instrumentation setup for the far-field acoustic and flow measurements, which were both performed in an aeroacoustic facility at Brunel University London that consists of an open jet wind tunnel and an anechoic chamber of $4 \mathrm{~m} \times 5 \mathrm{~m} \times 3.4 \mathrm{~m}$. The dimension of the open jet nozzle is 0.3 $\mathrm{m} \times 0.1 \mathrm{~m}$ (width $\times$ height). The aerofoil was attached to the side plate flush to the nozzle lips. Unless otherwise stated, all the far-field noise measurements, as well as the flow measurements, were performed at $U_{\infty}=24 \mathrm{~ms}^{-1}$. The open jet wind tunnel can produce very low turbulence intensity of $0.1 \%-0.2 \%$ between $10 \mathrm{~ms}^{-1}$ and $30 \mathrm{~ms}^{-1}$. The background noise (without the presence of the aerofoil, but with the side-plate) is largely contributed by the low subsonic jet noise, which is very low in comparison to the aerofoil self-noise level produced at the identical flow speed. All the far-field noise and flow measurements were performed at the geometric angle of attack $\theta=0^{\circ}$.

\section{Far-Field Noise Measurements}

The far-field noise measurements were performed with a $1 / 2$-inch condenser microphone mounted at a polar angle of $\Theta=90^{\circ}$, at the mid-span of the aerofoil and at $1 \mathrm{~m}$ above the trailing edge. The data were acquired using a 16-bit analogue-digital card manufactured by National Instrument. The sampling frequency was $44 \mathrm{kHz}$ with a sampling time of 20 seconds. The data were windowed and the Power Spectral Density (PSD) of $1 \mathrm{~Hz}$ bandwidth was computed from a 1024 point FFT and a 50\% overlap time. As stated in Section 2.2, noise measurements were performed for all the trailing edge cases at $U_{\infty}=24 \mathrm{~ms}^{-1}$.

\section{Near-Field Flow Measurements}

The near-field wake measurements were performed with a DANTEC miniature single hot wire straight probe (55P11). The probe was mounted parallel to the flow, and was positioned at $1 \mathrm{~mm}$ from the serration peaks. The hot wire measured the fluctuation and mean velocity of the aerofoil wake with an over-heat ratio set at 1.8. The hot wire signals were digitised by a 12-bit A/D convector (ADCPCI) at a sampling frequency of $20 \mathrm{kHz}$, through a DANTEC Dynamic Multichannel Constant Temperature Anemometer 54N80. The hot wire readings were recorded through ThermoPro software by TSI Inc. The hot wire probe was mounted to a two-dimensional traverse mechanism with a resolution of $0.01 \mathrm{~mm}$ in both directions. For all the serrated trailing edge cases, the flow measurements produced a series of two-dimensional wake profiles in the $y-z$ plane at $x=1 \mathrm{~mm}$ downstream of the peak of the serration. For 
the baseline B case, the wake measurement was at the same streamwise location as the serrated cases, which entails $\mathrm{x}=16 \mathrm{~mm}$ downstream of the straight trailing edge (therefore the wake profile will be more developed than the serrated cases). For the B, SS, SFS and MFS cases, the resolution in the $y$-direction is typically $0.1 \mathrm{~mm}$ in order to account for the large velocity gradient at the viscous sublayer region, but the resolution will gradually be relaxed as moving towards the freestream region. The resolution in the $z$-direction is consistent at $0.33 \mathrm{~mm}$ for a total distance of $9.9 \mathrm{~mm}$. This means that the spanwise measurements will cover four serration peaks and three serration troughs. For the SWS case, the spanwise wavelength $\lambda^{\prime}=30 \mathrm{~mm}$. This represents a challenging task to cover the full spanwise wavelength while keeping the same vertical and spanwise resolutions as the others. To save time, it was decided that only half of the spanwise wavelength will be measured, although six serration peaks and five serration troughs will still be covered.

\section{Results and Discussion}

The experimental noise results of the trailing edge serrations (SS, IFS, MFS, SFS and SWS), as well as the baseline (B) at $U_{\infty}=24 \mathrm{~ms}^{-1}$ will be presented in Section 3.1. Section 3.2 will discuss the flow behaviors of the nearwake produced by the above trailing edge devices.

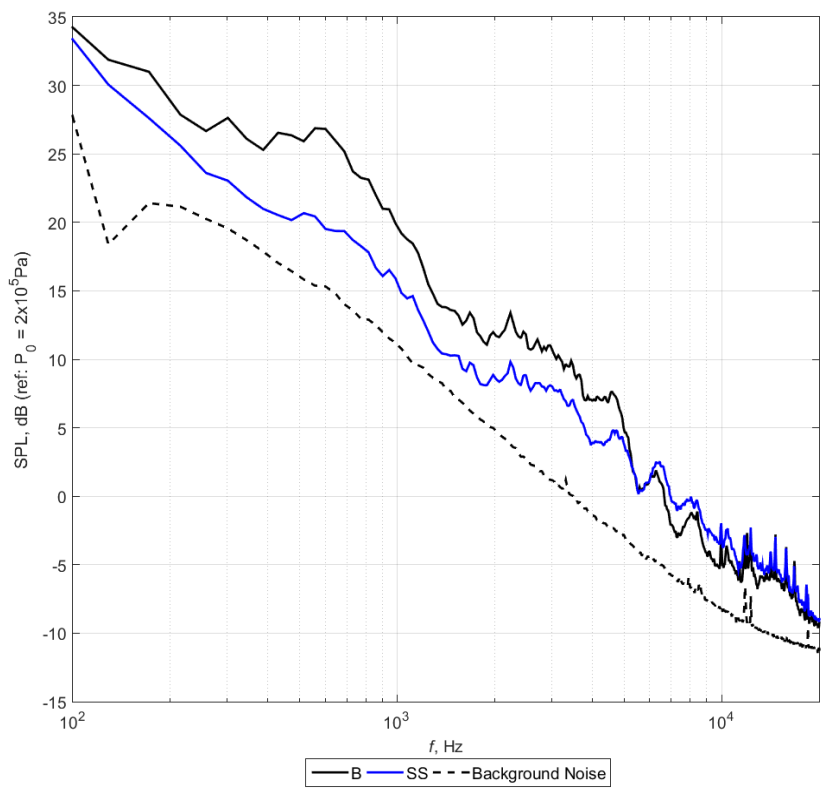

Fig 2 Comparison of the SPL, dB between the B, SS and background at $U_{\infty}=24 \mathrm{~ms}^{-1}$ and $\theta=0^{\circ}$.

\section{A. Noise Measurements}

A comparison of the aerofoil self-noise against the background noise of the open jet wind tunnel is shown in Figure 2. Similar to the study by Vathylakis et al. [12] who used the same facility, the background noise from the freestream flow (i.e. without the presence of the aerofoil) is shown to be significantly lower than the quiet self-noise produced by the aerofoil with SS. It is worth mentioning that the SPL spectrum produced by the SS case is lower than that by the B case over a significant portion of the low-to-mid frequency region. However, noise increase occurs at $f>6 \mathrm{kHz}$. The same characteristics of the self-noise radiation by the SS-type trailing edge shown here are also reported in the previous studies by León et al. [17], Vathylakis et al. [12], Gruber et al. [8], Oerlemans et al. [2] and Dassen et al. [19]. 


\section{B. Noise spectra of the sIngle-Flapped-Serration (IFS)}

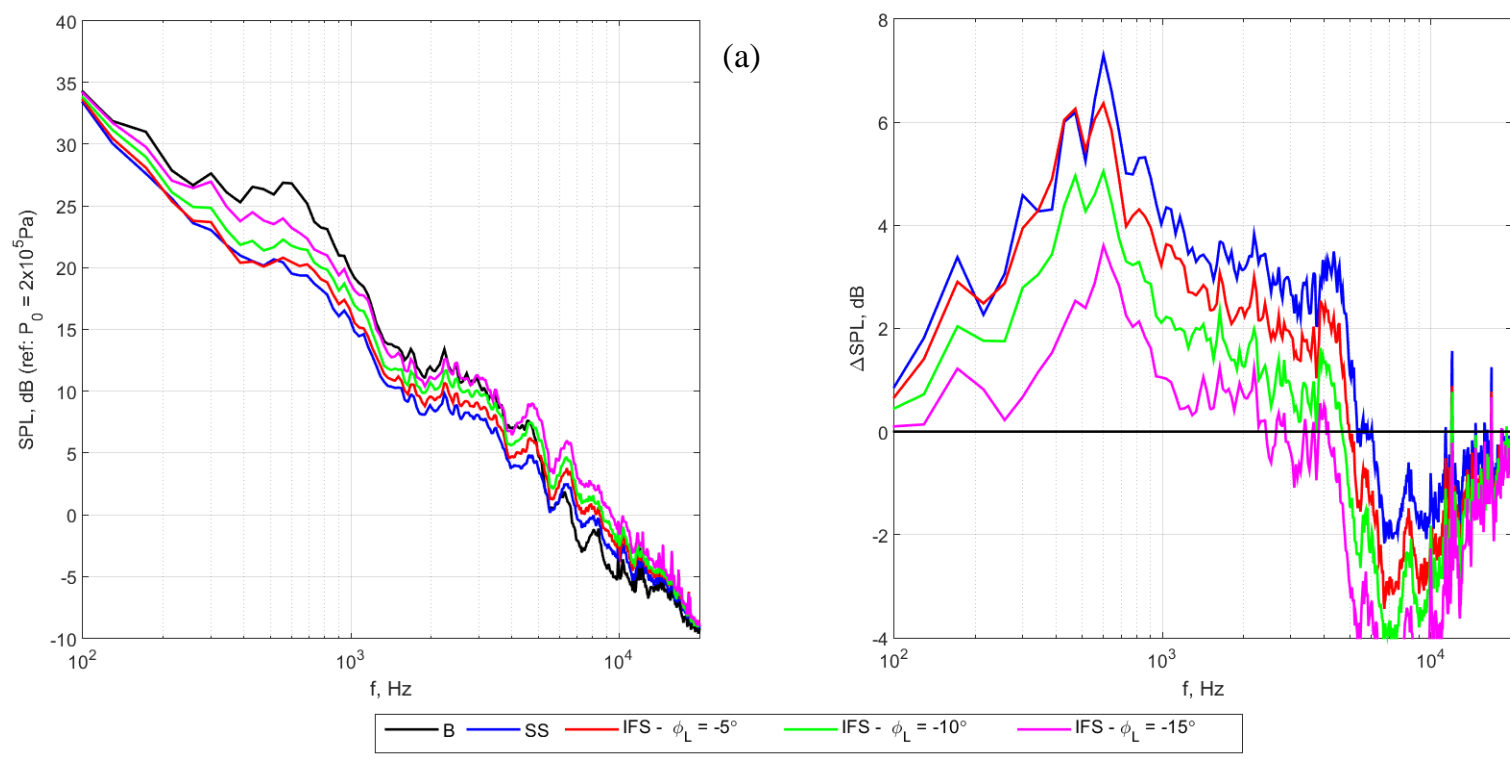

(b)

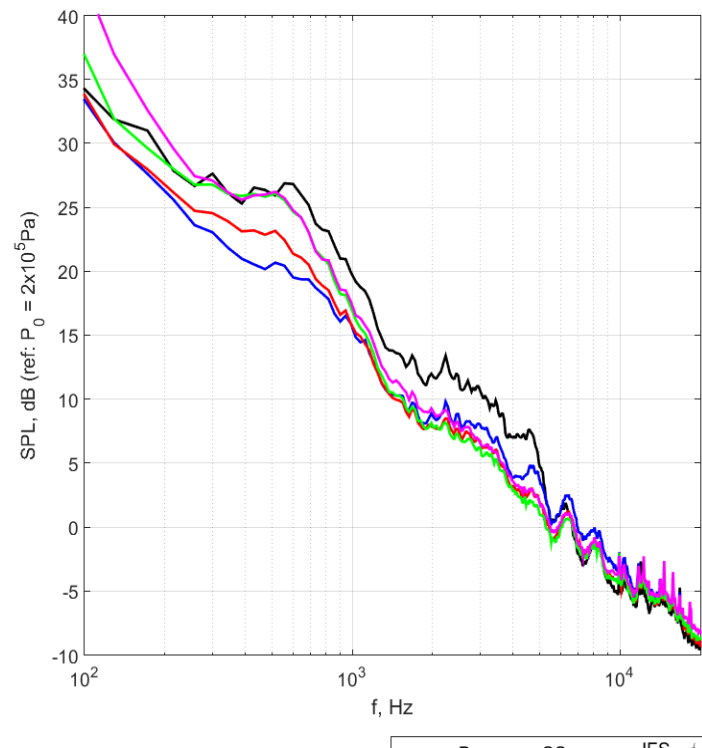

(c)

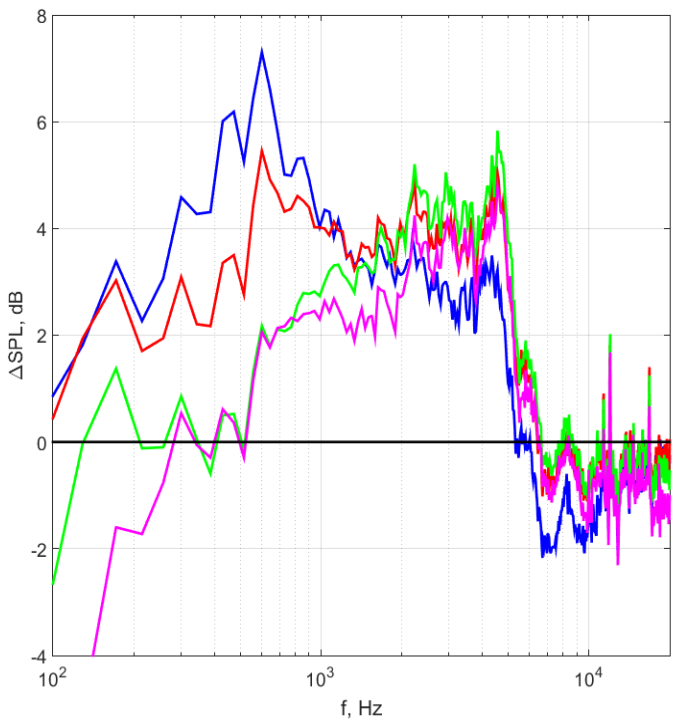

(d)

Fig 3 Comparison between the B, SS and IFS (different $\phi_{U}$ and $\phi_{L}$ ) trailing edges for: (a), (c) SPL, dB, and (b), (d) $\triangle$ SPL, dB. $U_{\infty}=24 \mathrm{~ms}^{-1}$ and $\theta=0^{\circ}$.

Figure 3 shows the sensitivities of the broadband noise radiation to the SS and IFS-type trailing edges. $\triangle$ SPL is defined as the difference in the sound pressure level, as a function of frequency, between the baseline and serrated trailing edges: $\Delta \operatorname{SPL}(f)=\operatorname{SPL}_{\mathrm{B}}(f)-\operatorname{SPL}$ SS, or IFS $(f)$. Note that a positive value of $\Delta$ SPL represents noise reduction, and the opposite is true. This definition also applies to the other serrated trailing edge cases to be discussed later. For the SPL IFS case, the $\phi_{U}$ and $\phi_{L}$ denote the flap-up and flap-down angles, respectively. The results in Fig. 3 in the current study follow largely the same trend as Vathylakis et al. [12]. Figure $3 \mathrm{~b}$ clearly demonstrates that the levels of broadband noise reduction ( $\triangle$ SPL) achieved by all the IFS, flap-down trailing edges are inferior than that achieved by the conventional SS trailing edge. The larger the flap-down angle of the serration, the lower the $\Delta$ SPL can be achieved. Moreover, a large flap-down angle will also cause higher level of noise increase at the high frequency region. 
On the other hand, the IFS flap-up configurations produce better positive outlook in comparison to the SS trailing edge (Fig. 3d). The $\triangle$ SPL achieved by the IFS flap-down configurations are normally less than that by the SS trailing edge at typically $f<1.5 \mathrm{kHz}$. However, above this frequency, all the IFS flap-down trailing edges outperform the SS trailing edge. For example, the $\triangle \mathrm{SPL}(f=4.5 \mathrm{kHz})$ achieved by the IFS $\phi_{U}=10^{\circ}$ and the SS trailing edges are $5.8 \mathrm{~dB}$ and $3.0 \mathrm{~dB}$, respectively. In addition, all the IFS for flap-up configurations already produce lower level of negative $\Delta$ SPL (i.e. noise increase over the baseline trailing edge) than that by the SS trailing edge. Overall, the most promising candidate for the IFS flap-up configuration is the $\phi_{U}=5^{\circ}$ case. The consistently high level of $\Delta \mathrm{SPL}$ at $f>1.5 \mathrm{kHz}$ achieved by this type of configuration warrants further efforts to improve the noise performance at $f<1.5 \mathrm{kHz}$ by exploiting different concepts of the serration misalignment. The results will be discussed in the subsequent sub-sections.

\section{Noise spectra of the Multi-Flapped-Serration (MFS)}
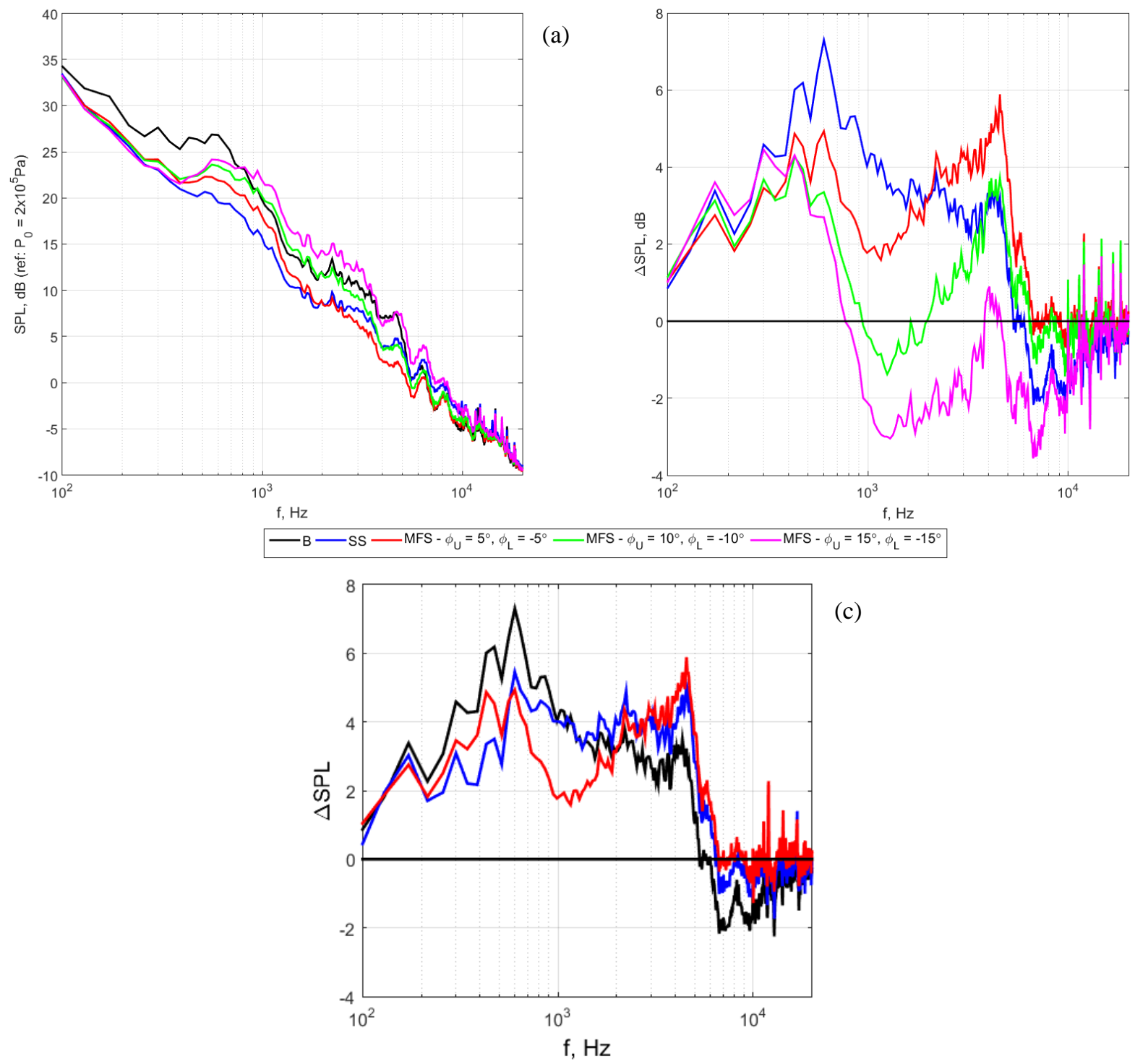

(c)

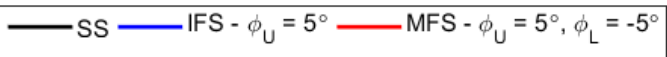

Fig 4 Comparison between the B, SS, IFS and MFS $\left(\left|\phi_{U}\right|=\left|\phi_{L}\right|\right)$ trailing edges for: (a) SPL, dB, (b) $\Delta S P L$, $\mathrm{dB}$, and (c) $\Delta \mathrm{SPL}, \mathrm{dB} . U_{\infty}=24 \mathrm{~ms}^{-1}$ and $\theta=0^{\circ}$. 
Figure 4 shows the sensitivities of the broadband noise radiation to the SS and MFS-type trailing edges. For the MFS presented in Fig. 4, the $\left|\phi_{U}\right|$ is equal to $\left|\phi_{L}\right|$. The $\Delta$ SPL spectra in Fig. 4b show that the best performer amongst the MFS trailing edges is when $\left|\phi_{U}\right|=\left|\phi_{L}\right|=5^{\circ}$. The overall acoustic performance reduced with increases of both $\left|\phi_{U}\right|$ and $\left|\phi_{L}\right|$. When the IFS flap-up $\left|\phi_{U}\right|=5^{\circ}$ is compared against the MFS $\left|\phi_{U}\right|=\left|\phi_{L}\right|=5^{\circ}$, as well as the SS (i.e. $\phi_{U}=\phi_{L}=0^{\circ}$ ) trailing edges in Fig. $4 \mathrm{c}$, the followings can be observed:

1.At $f<600 \mathrm{~Hz}$, the $\Delta$ SPL achieved by the MFS is slightly better than the IFS counterpart, but worse than the SS trailing edge.

2. At $600 \mathrm{~Hz}<f<2.8 \mathrm{kHz}$, there is a degradation in $\triangle \mathrm{SPL}$ by the MFS in relation to the IFS trailing edge, but it has already outperformed the SS trailing edge as early as $f=2 \mathrm{kHz}$.

3. At $2.8 \mathrm{kHz}<f<6.7 \mathrm{kHz}$, the MFS consistently outperforms the IFS and SS trailing edges and achieves higher $\Delta$ SPL. The largest noise reduction is achieved by the MFS case at $\Delta \mathrm{SPL}=6 \mathrm{~dB}$ at $f=4.6 \mathrm{kHz}$.

At $f>6.7 \mathrm{kHz}$, both the IFS and SS trailing edges experience negative level of $\Delta$ SPL (i.e. noise increase). However, the MFS averaged at $\Delta \mathrm{SPL} \approx 0$ throughout this high frequency region. This implies that a periodic misalignment of the serrated trailing edge could reduce the cross-flow component in the space between adjacent members of the serration. This point will be discussed in Section 3.2.

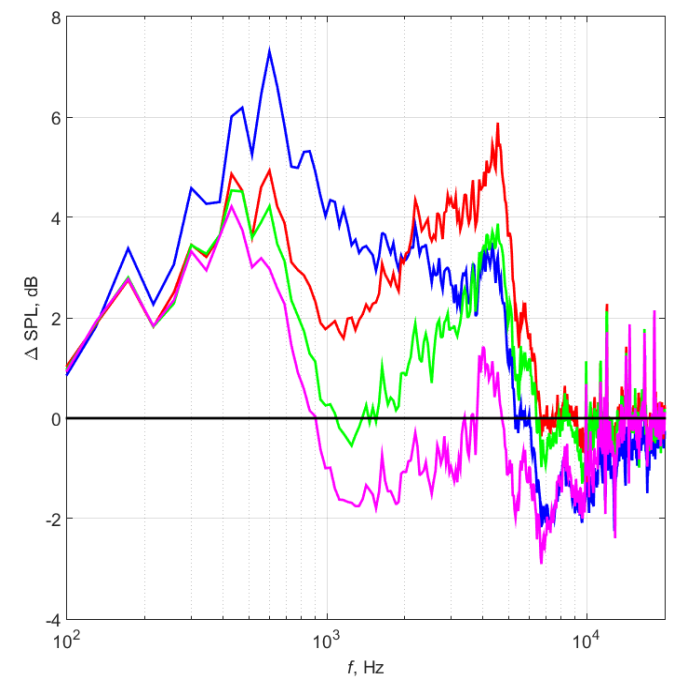

(a)

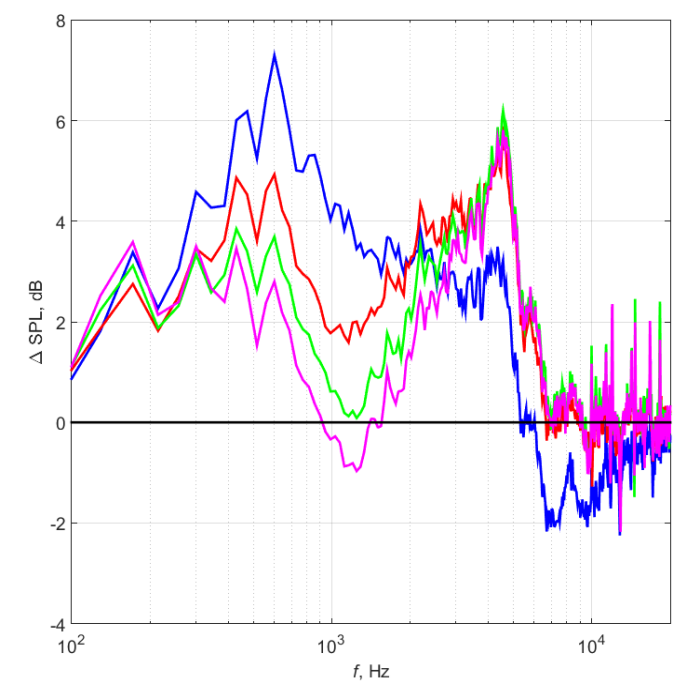

(b)

Fig 5 Comparison of the $\triangle$ SPL produced by the SS and MFS trailing edges for: (a) $\phi_{U}=5^{\circ}, \phi_{L}=-5^{\circ},-10^{\circ} \&$ $15^{\circ}$, and (b) $\phi_{U}=5^{\circ}, 10^{\circ} \& 15^{\circ}, \phi_{L}=-5^{\circ} . U_{\infty}=24 \mathrm{~ms}^{-1}$ and $\theta=0^{\circ}$.

The MFS $\left|\phi_{U}\right|$ and $\left|\phi_{L}\right|$ have shown to be advantageous when both are at $5^{\circ}$. The next investigation is to examine the $\Delta$ SPL spectra when $\left|\phi_{U}\right|=5^{\circ}$ and $5^{\circ}<\left|\phi_{L}\right|<15^{\circ}$. As shown in Fig. 5a, the results are consistent with the IFS trailing edges in Fig. $3 b$ in that increasing the flap-down angle will cause a degradation of the noise reduction performance. On the other hand, for the $\triangle$ SPL spectra achieved by the MFS trailing edge with $5^{\circ}<\left|\phi_{U}\right|<$ $15^{\circ}$ and $\left|\phi_{L}\right|=5^{\circ}$ in Fig. 5 b:

1. At $f<600 \mathrm{~Hz}$, the curves demonstrate a markedly improvement of the $\Delta$ SPL when compared to the corresponding IFS trailing edges in Fig. $3 \mathrm{~d}$ for $5^{\circ} \leq\left|\phi_{U}\right| 15^{\circ}$, but $\left|\phi_{L}\right|=0^{\circ}$.

2. At $600 \mathrm{~Hz}<f<2-2.8 \mathrm{kHz}$, there is a degradation in $\square$ SPL by the MFS in relation to the SS trailing edge. It is clear that the higher the $\left|\phi_{U}\right|$, the larger level of the degradation in the $\Delta$ SPL.

3. At $2.8 \mathrm{kHz}<f<6.7 \mathrm{kHz}$, a collapse of the $\Delta \mathrm{SPL}$ curves has been demonstrated regardless the value of the $\left|\phi_{U}\right|$. This is also consistent with the corresponding IFS trailing edges in Fig. 3d. Most importantly, and perhaps also counter-intuitively, it seems that having a small flap-down $\left|\phi_{L}\right|$ angle on a MFS 
configuration could actually improve the range of $\left|\phi_{U}\right|$ under which higher $\Delta$ SPL can be achieved (compare Fig. 5b and Fig. 3d).

4. At $\mathrm{f}>6.7 \mathrm{kHz}$, all the MFS trailing edges averaged at $\triangle \mathrm{SPL} \approx 0$ throughout this high frequency region. This also implies that setting a small $\left|\phi_{L}\right|$ on the MFS configuration could be beneficial.

Overall, the results presented in this sub-section suggest that it is possible to fine-tune both the $\left|\phi_{U}\right|$ and $\left|\phi_{L}\right|$ of a MFS trailing edge to improve the level of $\triangle$ SPL. The results indicate that a small $\left|\phi_{L}\right|$ is beneficial. It has also been shown that the noise improvement is versatile across the whole range of frequency $(100 \mathrm{~Hz} \leq \mathrm{f} \leq 20 \mathrm{kHz})$.

\section{Noise spectra of the Split-Flapped-Serration (SFS)}

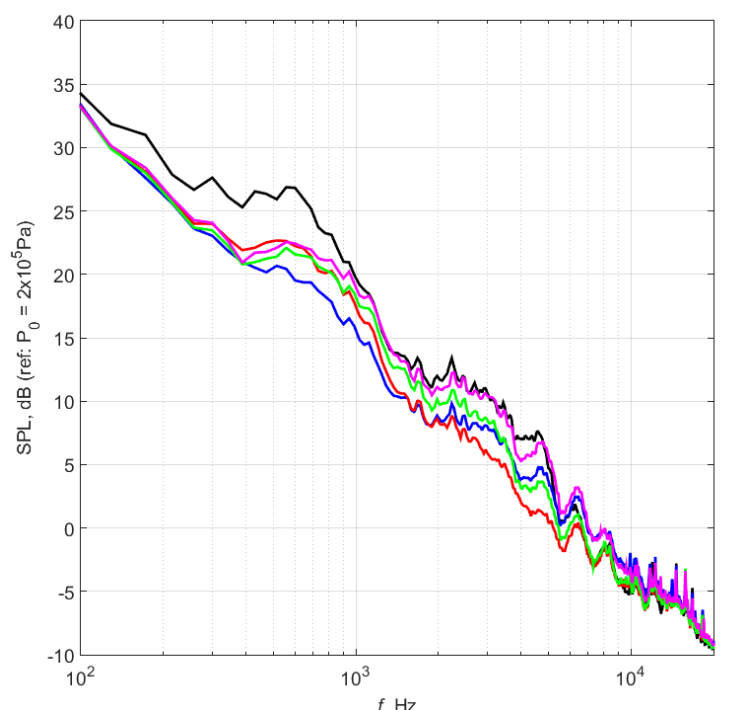

(a)

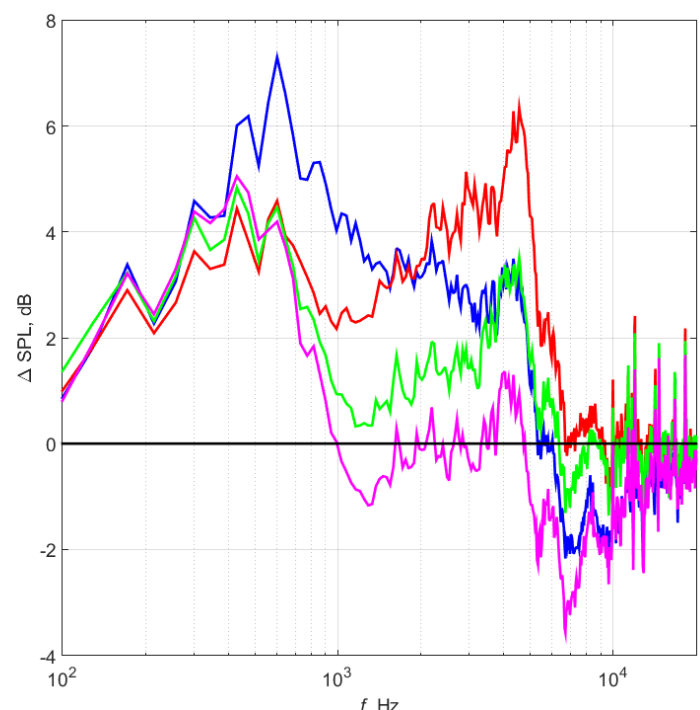

(b)
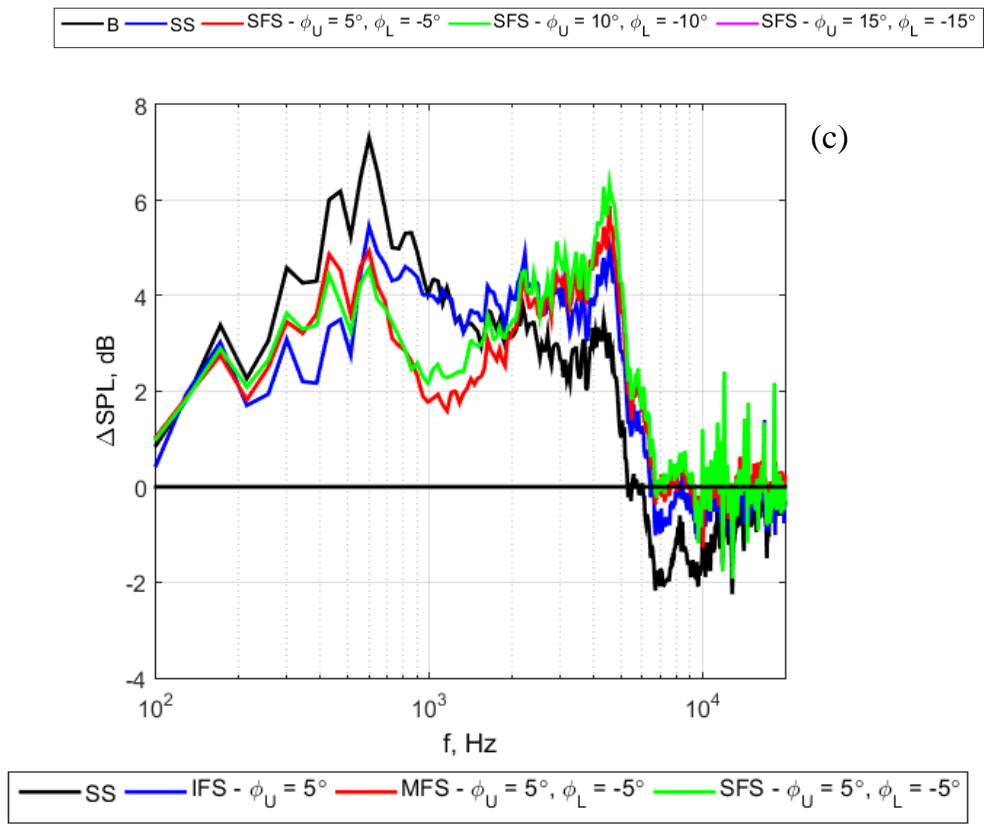

Fig 6 Comparison between the B, SS and SFS $\left(\left|\phi_{U}\right|=\left|\phi_{L}\right|\right)$ trailing edges for: (a) SPL, dB, (b) $\Delta$ SPL, dB, and (c) $\triangle \mathrm{SPL}, \mathrm{dB} . U_{\infty}=24 \mathrm{~ms}^{-1}$ and $\theta=0^{\circ}$. 
Figures $6 \mathrm{a}$ and $6 \mathrm{~b}$ show the sensitivities of the broadband noise radiation to the SS and SFS-type trailing edges. Generally speaking, the noise charactristics and trends discussed in Section 3.1.2 for the MFS trailing edges are largely applicable to the SFS trailing edges, although the absolute level of $\triangle$ SPL achieved by the SFS trailing edge is generally larger than the MFS trailing edge, as shown in Fig. 6c. For example, the MFS $\left|\phi_{U}\right|=\left|\phi_{L}\right|=5^{\circ}$ trailing edge achieved the largest $\Delta \mathrm{SPL}=6 \mathrm{~dB}$ at $f=4.6 \mathrm{kHz}$. At the same frequency, the $\Delta \mathrm{SPL}$ achieved by the SFS $\left|\phi_{U}\right|$ $=\left|\phi_{L}\right|=5^{\circ}$ trailing edge is $6.5 \mathrm{~dB}$. The advantages of the SFS trailing edges over the MFS are further manifested when compare between the SFS $\left|\phi_{U}\right|=\left|\phi_{L}\right|=15^{\circ}$ and the MFS $\left|\phi_{U}\right|=\left|\phi_{L}\right|=15^{\circ}$ (Fig. 6b vs. Fig. 4b, respectively).

The $\Delta$ SPL spectra when SFS $\left|\phi_{U}\right|=5^{\circ}$ and $5^{\circ} \leq\left|\phi_{L}\right| \leq 15^{\circ}$ is shown in Fig. 7a. Figure $7 \mathrm{~b}$ shows the $\Delta$ SPL spectra when SFS $\left|\phi_{L}\right|=5^{\circ}$ and $5^{\circ} \leq\left|\phi_{U}\right| \leq 15^{\circ}$. The noise characteristics and trends are again similar to the MFS trailing edges (compare Fig. 7 and Fig. 5), although there are also many instances the SFS trailing edges can achieve a higher $\triangle$ SPL than the MFS.
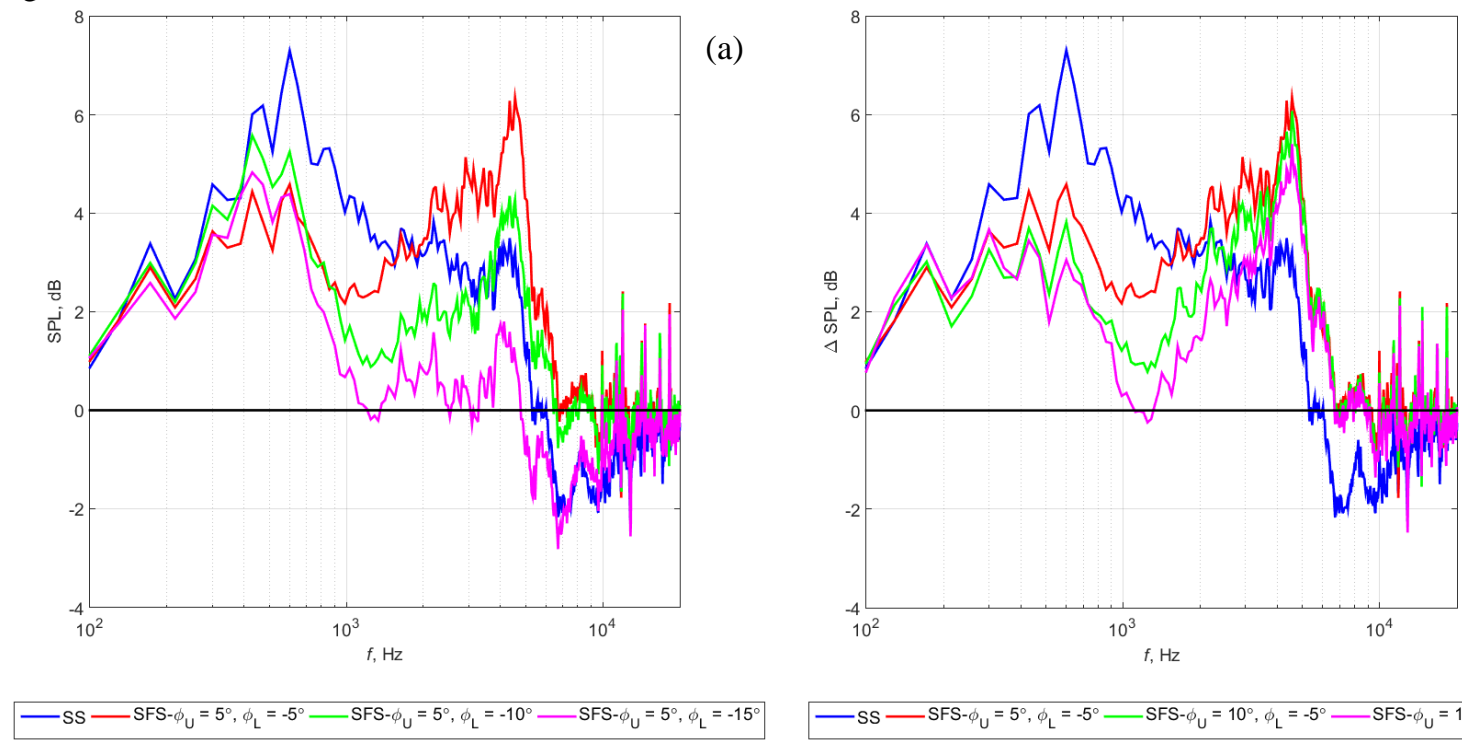

(b)

Fig 7 Comparison of the $\triangle$ SPL produced by the SS and SFS trailing edges for: (a) $\phi_{U}=5^{\circ}, \phi_{L}=-5^{\circ},-10^{\circ} \&$ $15^{\circ}$, and (b) $\phi_{U}=5^{\circ}, 10^{\circ} \& 15^{\circ}, \phi_{L}=-5^{\circ} . U_{\infty}=24 \mathrm{~ms}^{-1}$ and $\theta=0^{\circ}$.

\section{E. Noise spectra of the Spanwise-Wavy-Serration (SWS)}

So far, the best candidate for the low-noise trailing edge device is the Split-Flapped-Serration (SFS). However, just like the MFS and IFS counterparts, they all exhibit a drop of the $\Delta$ SPL between $300 \mathrm{~Hz}<f<2.4 \mathrm{kHz}$ when compared to the conventional serration of the SS trailing edge (note that the stated frequency range is averaged across the scatter of the available dataset). The IFS, MFS and SFS trailing edge devices all feature a common characteristic in that the rate of change of the flap angle across the spanwise direction $d \phi / d z$, is either zero (IFS) or very large (MFS and SFS). It is assumed that a more gentle value of the $d \phi / d z$ might reduce the excessive strain of the secondary flow near the trailing edge, and this represents another optimisation parameter for the out-of-phase trailing edge serration which will be discussed here.

Figure 8 shows the sensitivities of the broadband noise radiation to the SS and SWS-type trailing edges of different $\lambda^{\prime}$, but all with $\left|\phi_{U}\right|=\left|\phi_{L}\right|=10^{\circ}$. It is very clear from the figure that, at $300 \mathrm{~Hz}<f<2.4 \mathrm{kHz}$ where the noise performance suffers a degradation for the IFS, MFS and SFS trailing edges, no longer exists for the SWS trailing edge. However, the $\triangle \mathrm{SPL}$ achieved by the SWS trailing edge at the high frequency is generally less than those by the MFS and SFS. For example, the largest $\Delta$ SPL at $f=4.6 \mathrm{kHz}$ achieved by the MFS $\left|\phi_{U}\right|=\left|\phi_{L}\right|=5^{\circ}$ and SFS $\left|\phi_{U}\right|=\left|\phi_{L}\right|=5^{\circ}$ are $6 \mathrm{~dB}$ and $6.5 \mathrm{~dB}$, respectively. However, the corresponding $\triangle$ SPL achieved by the SWS $\lambda^{\prime}=15 \mathrm{~mm},\left|\phi_{U}\right|=\left|\phi_{L}\right|=5^{\circ}$ trailing edge is $5 \mathrm{~dB}$. Overall, there is also no much variation in the acoustic performance against the different values of $\lambda^{\prime}$. It can be regarded that the $\lambda^{\prime}=15 \mathrm{~mm}$ case performs better than the larger $\lambda^{\prime}$ cases. 
Figure 9 compares the $\triangle$ SPL spectra produced by the SS and SWS trailing edges. The SWS trailing edges have the same $\lambda^{\prime}=45 \mathrm{~mm}$, but with $5^{\circ} \leq\left|\phi_{U}\right|,\left|\phi_{L}\right| \leq 15^{\circ}$. Note that in the current paper both the $\left|\phi_{U}\right|$ and $\left|\phi_{L}\right|$ for the SWS trailing edge are kept the same. It can be demonstrated that the overall acoustic performance of the SWS trailing edge is reduced when the flap angle increases. Based on the observations in Fig. 8 and Fig. 9, the best reduction in the broadband noise level for the SWS trailing edge is therefore associated with both small $\phi$ and $\lambda^{\prime}$.

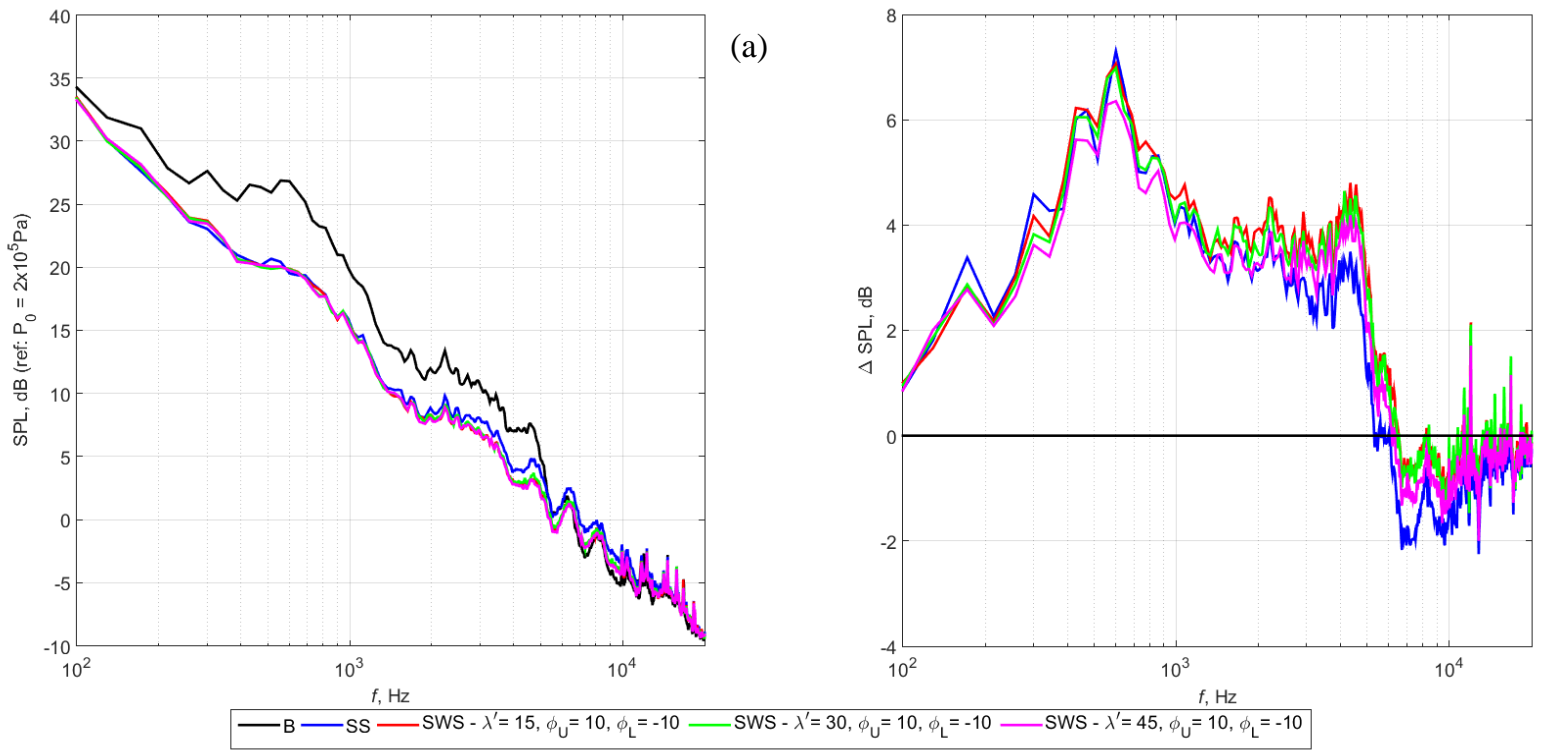

Fig 8 Comparison between the B, SS and SWS $\left(\lambda^{\prime}=15,30 \& 40 \mathrm{~mm},\left|\phi_{U}\right|=\left|\phi_{L}\right|\right)$ trailing edges for: (a) SPL, dB, and (b) $\triangle$ SPL, dB. $U_{\infty}=24 \mathrm{~ms}^{-1}$ and $\theta=0^{\circ}$.

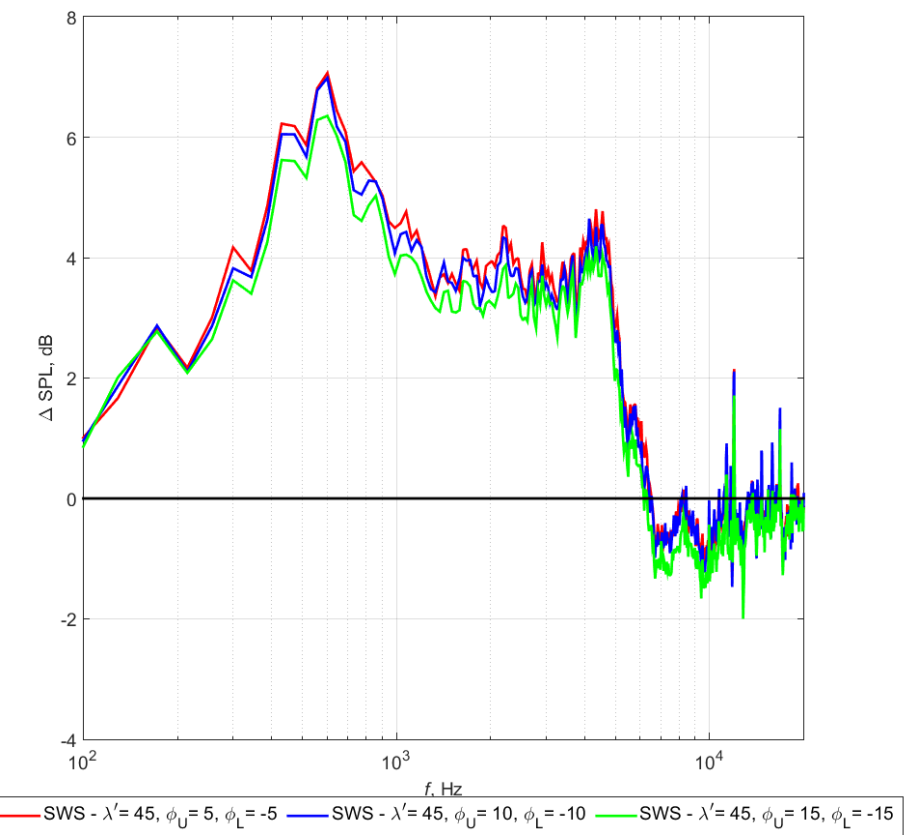

Fig 9 Comparison of the $\triangle$ SPL produced by the SS and SWS trailing edges for $\lambda^{\prime}=45 \mathrm{~mm}$, and $\left|\phi_{U}\right|=\left|\phi_{L}\right|=$ $5^{\circ}, 10^{\circ}$ and $15^{\circ} . U_{\infty}=24 \mathrm{~ms}^{-1}$ and $\theta=0^{\circ}$. 


\section{F. Comparison of all the trailing edge devices for their noise performances}

Summary of the acoustic performance of the four trailing edge devices, SS, MFS, SFS and SWS, is shown in Figure 10. It is clear that all the "misaligned" trailing edges (MFS, SFS and SWS) produce different noise characteristics compared to the conventional SS trailing edge. The MFS and SFS trailing edges are largely similar in the $\Delta$ SPL spectra, although the SFS achieves slightly larger level of the $\Delta$ SPL. At $f<2.4 \mathrm{kHz}$, both the MFS and SFS still achieve noise reduction over the baseline B trailing edge, but the level of noise reduction is less than the conventional SS trailing edge. However, between $2.4 \mathrm{kHz}<f<6.7 \mathrm{kHz}$, a reverse situation occurs in that they now outperform the SS trailing edge considerably. At $f>6.7 \mathrm{kHz}$, where both the IFS and SS trailing edges would otherwise experience negative level of $\Delta$ SPL (i.e. noise increase), both the MFS and IFS averaged at $\Delta \mathrm{SPL} \approx 0$ throughout this high frequency region. This implies that a periodic misalignment of the serrated trailing edge could reduce the cross-flow component in the space between adjacent members of the serration.

For the SWS trailing edge, somehow an opposite trend is observed. Unlike the MFS and SFS trailing edges, the SWS trailing edge produces the same level of $\Delta$ SPL as the SS trailing edge across the frequency range of $f<2.4$ $\mathrm{kHz}$. Between $2.4 \mathrm{kHz}<f<6.7 \mathrm{kHz}$, the SWS trailing edge still outperforms the SS trailing edge, but the level of $\triangle \mathrm{SPL}$ it can achieve is now less than those of MFS and SFS trailing edges. At $f>6.7 \mathrm{kHz}$, the SWS begins to experience negative $\triangle$ SPL, indicating a noise increase over the baseline B trailing edge. However, the increase in noise level by the SWS trailing edge at this high frequency range is still less than that by the SS trailing edge.

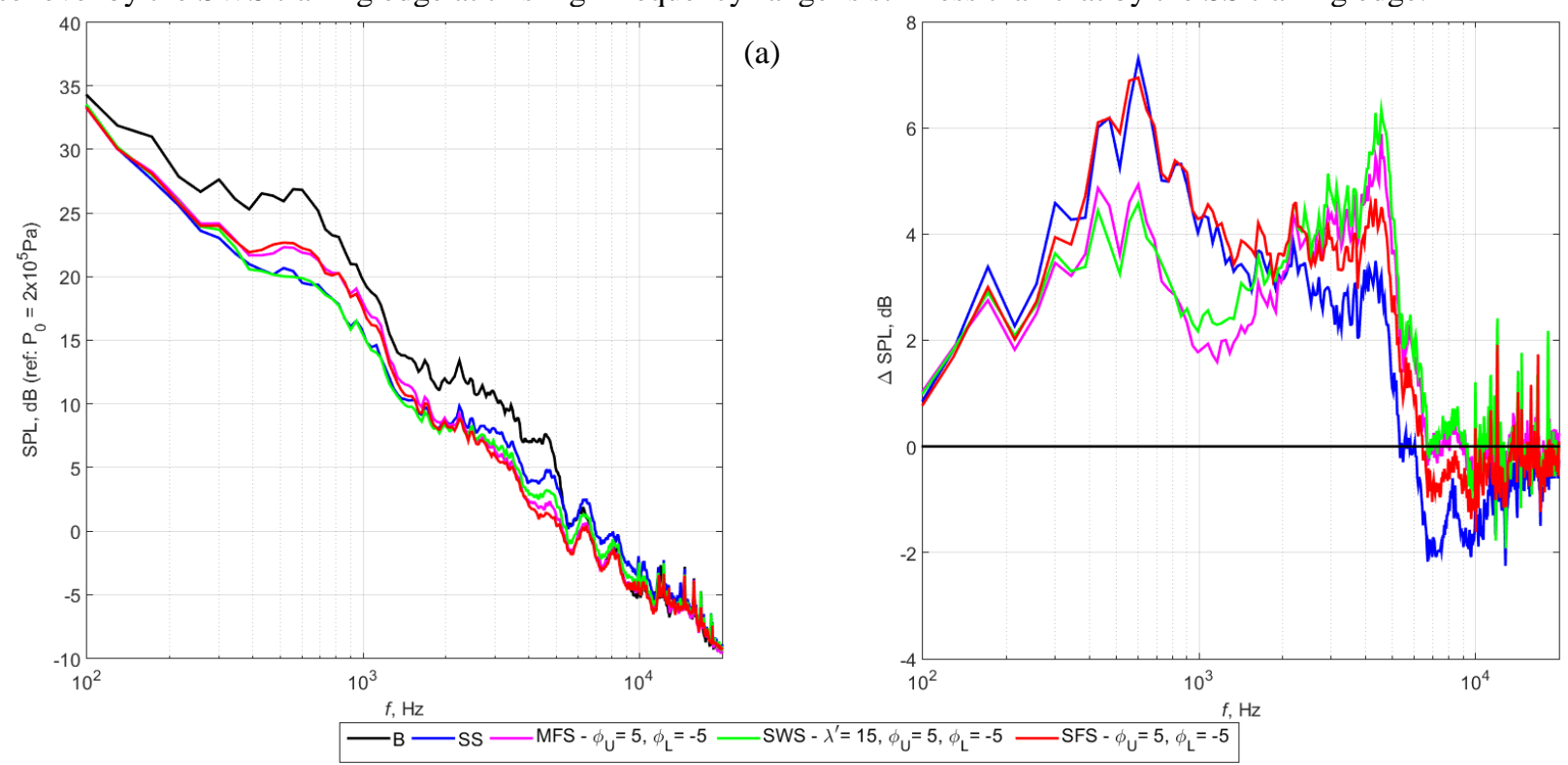

Fig 10 Comparison between the B, SS, MFS, SFS and SWS trailing edges for: (a) SPL, dB, and (b) $\triangle$ SPL, dB. $U_{\infty}=24 \mathrm{~ms}^{-1}$ and $\theta=0^{\circ}$.

\section{G. Near-Wake Flow Behaviors}

The wake flow across the serrated and baseline trailing edges were investigated at $1 \mathrm{~mm}$ and $16 \mathrm{~mm}$ downstream of the trailing edge, respectively, in the $y-z$ plane. These were performed in the same open-jet aeroacoustic wind tunnel using a single hot-wire probe. The B, SS, MFS $\left|\phi_{U}\right|=\left|\phi_{L}\right|=5^{\circ}$, SFS $\left|\phi_{U}\right|=\left|\phi_{L}\right|=5^{\circ}$ and SWS $\lambda^{\prime}=45 \mathrm{~mm}$, $\left|\phi_{U}\right|=\left|\phi_{L}\right|=5^{\circ}$ were investigated. The turbulence intensity $\left(u_{r m s} / U_{\infty}\right)$ contour maps are presented in Fig. 11 .

The first major observation is that the size of the turbulent wake on the pressure side (i.e. $y<0$ ) is significantly larger than the wake region on the suction side (i.e. $y>0$ ) for all cases. This implies that the boundary layer near the trailing edge on the suction side is thinner than that on the pressure side. Vathylakis et al. (2016) also observed the same trend, and they explained that this might be due to the NACA65(12)-10 being originally designated as a laminar aerofoil. Because the thickest part of the aerofoil occurs at a considerable distance downstream of the leading edge (see Fig. 1), the growth of boundary layer on the suction side will experience a considerable level of acceleration over the first half of the aerofoil. After that the adverse pressure gradient takes over where the flow will be decelerated towards the trailing edge. On the other hand, the pressure side is largely flat but exhibits a constant expansion angle. The large level of adverse pressure gradient over the entire chord of the pressure side will encourage a considerable thickening of the boundary layer by the time it reaches the trailing edge. 
From Fig. 11, there is little variation of the turbulence intensity level across the spanwise distance for the baseline B trailing edge. Note that $y=0$ represents the trailing edge. The near wake is characterised by low turbulence level near the trailing edge as it is related to the viscous, inner part of the upstream boundary layers on both the suction and pressure sides. The maximum turbulence level is achieved at an intermediate vertical distance which is largely associated with the upstream buffer layer of the boundary layer. Finally, the turbulence intensity will gradually be reduced to very low level at the freestream. Note that this particular $y-z$ turbulence intensity contour map for the B trailing edge was measured at $x=16 \mathrm{~mm}$ from the trailing edge.

The serration amplitudes for the IFS, MFS, SFS and SWS are the same at $2 h=30 \mathrm{~mm}$. In order to follow the standard practice of matching the same wetted area, the amplitude of the B trailing edge was chosen as $15 \mathrm{~mm}$. For all the serrated trailing edges, their $y-z$ turbulence intensity contour maps were measured at exactly the same streamwise location as the baseline B trailing edge. This means that during the changeover of the different trailing edge devices, the hot wire probe always remained at the same streamwise location. Therefore, the distance between the hot wire measurement point and the B trailing edge is $16 \mathrm{~mm}$. For the IFS, MFS, SFS and SWS cases, the distance between the hot wire measurement point and the serration peak and serration trough is $1 \mathrm{~mm}$ and $31 \mathrm{~mm}$, respectively.

Based on the above arrangement, one would expect that the "area" of the turbulent wake region for the B trailing edge, which can be defined by the turbulence intensity to some extent, will be rather similar to the turbulent wake area somewhere in between the serration peak and trough for the serrated case. Interestingly, this is not the case. The $y-z$ turbulence intensity contour maps produced by the SS trailing edge exhibits the followings:

1. Thickening of the turbulent wake on the suction side, but thinning on the pressure side. The overall thickness of the turbulent wake, however, remains roughly the same compared to the B trailing edge case.

2. The dominant turbulence intensity region associated with the suction side buffer layer has been displaced upward.

3. The presence of an $\cap$ shape high turbulence intensity structure at every sawtooth gap.

4. High turbulence intensity on the pressure side at the serration peak region.

Combining the four points above, a hint about the mechanism of the broadband noise reduction and high frequency noise increase by the conventional SS trailing edge can be discerned. That is, the sawtooth gap represents a leakage mechanism to draw cross-flow from the pressure side to the suction side through the sawtooth gaps. The fluid-structure interaction between the cross-flow and the serration then increases the local turbulence level and inevitably produces an extraneous noise (i.e. the high frequency noise increase). However, these cross-flow components could also trigger a global displace of the turbulent wake, or the upstream boundary layer, such that the dominant turbulence production mechanism on the suction side is now far from the wall. At the same time, there is absence of a homogeneous buffer layer on the pressure side, partly due to the entrainment of the cross-flow component at the sawtooth gap, and partly due to the transfer of turbulent energy to form the high turbulence structure near the serration peak region as manifested in the $y-z$ contour map in Fig. 11. As a result, the previously effective mechanism for the turbulent broadband noise radiation by the straight trailing edge is now significantly disrupted by the SS trailing edge, which then leads to a reduction of the broadband noise.

For the MFS trailing edge, the flap-up sawteeth seem to be able to compartmentalise the suction side buffer layer into individual cell structure. This could represent an additional mechanism to provide a further broadband noise reduction as manifested in Fig. 10b. On the other hand, the turbulent wake within the flap-up and flap-down sawteeth is more complex as it might be formed by a combination of the cross-flow component and separated flow (due to the presence of flap angle). Nevertheless, such complex flow field could explain the very low noise increase at the high frequency region when compared to the SS trailing edge. The SFS trailing edge can indeed support this hypothesis because the turbulence intensity level at the turbulent wake region between the flap-up and flap-down sawteeth is becoming even less significant due to the similar, if not more complex flow field.

The $y-z$ turbulence intensity contour map produced by the SWS trailing edge in Fig. 11 only covers one-third of the spanwise wavy wavelength $\lambda^{\prime}$. Overall, all the characteristics described in points $1-4$ above pertaining to the SS trailing edge are also applicable to the SWS trailing edge, except that the individual $\cap$ structure is now skewed according to the angle of orientation of the corresponding sawtooth. These may explain the noise performance for the SWS trailing edge is not significantly different with the SS trailing edge at the low-to-mid, as well as the very high frequency regions (see Fig. 10b). However, the SWS could still achieve better broadband noise reduction level than the SS trailing edge across the mid-to-high frequency. This may be explained by the presence of the compartmentalisation of the buffer layer on the suction side by the SWS trailing edge. 

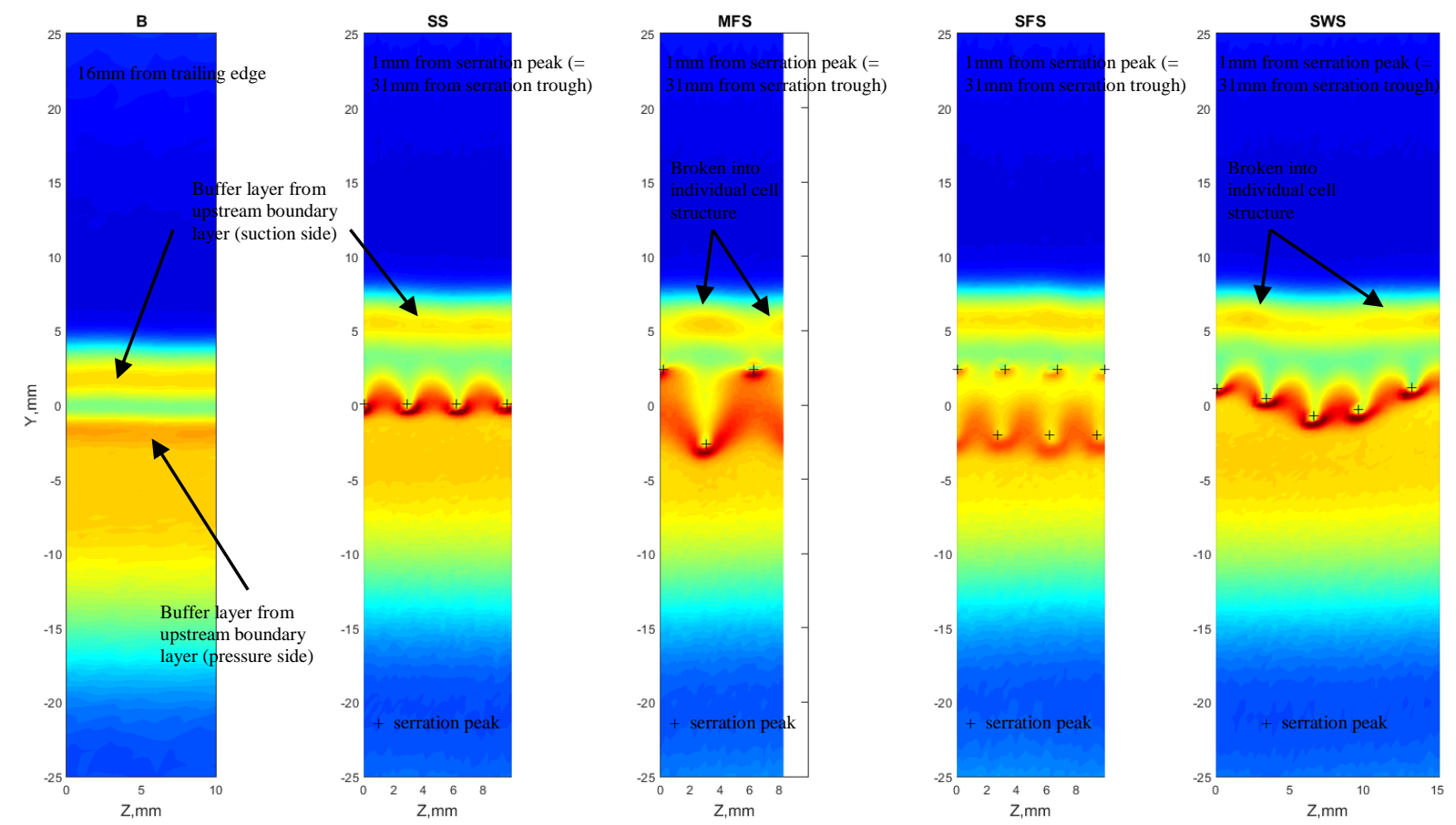

Fig 11 Turbulence intensity contour maps in the $y-z$ plane for the B, SS, MFS, SFS, and SWS trailing edges at $\mathrm{U}=\mathbf{2 4} \mathrm{ms}^{-1}$ and $\theta=0^{\circ}$.

\section{Conclusion}

This paper presents the experimental results of adding variable flap angles to the serrated trailing edges and their effects on the self-noise radiation of an aerofoil. The investigation included aeroacoustic and wake flow measurements on an NACA 65-(12)10 aerofoil. This paper explores further combinations of positive and negative serration flap angles, $\phi_{\mathrm{U}}$ and $\phi_{\mathrm{L}}$ respectively, across the spanwise extent of the trailing edge. A new parameter, the serration oscillation wavelength $\left(\lambda^{\prime}\right)$, was also included. The study investigates five individual cases, Baseline (B), Straight-Serration (SS), sIngle-Flapped-Serration (IFS), Multi-Flapped-Serration (MFS), Split-Flapped-Serration (SFS) and Spanwise-Wavy-Serration (SWS). The serration amplitude and wavelength remained consistent as $30 \mathrm{~mm}$ and $3.3 \mathrm{~mm}$ respectively. It is clear that all the "misaligned" trailing edges produce different noise characteristics compared to the conventional SS trailing edge. At low-to-mid frequency range, the conventional SS trailing edge still outperforms both the MFS and SFS trailing edges. However, from mid-to-high frequency, both the MFS and SFS trailing edges can achieve higher noise reduction than the SS trailing edge considerably. At very high frequency, where the SS trailing edge would otherwise experience noise increase, there is no noise increase for both the MFS and SFS trailing edges because the periodic misalignment reduces the cross-flow component in the space between adjacent members of the serration. The SWS trailing edge produces the same level of noise reduction as the SS trailing edge across the low-to-mid frequency range. From mid-to-high frequency range, the SWS trailing edge outperforms the SS trailing edge, but the level of further noise reduction it can achieve is less than those of MFS and SFS trailing edges. At very high frequency, the SWS begins to experience slightly noise increase over the baseline B trailing edge. However, the increase in noise level by the SWS trailing edge at this high frequency range is still less than that by the SS trailing edge. The near-wake flow measurement results provide some explanations for the mechanisms underpinning the broadband noise reduction and high frequency noise increase by these trailing edge devices. 


\section{Acknowledgments}

The authors would like to thank the financial support from the EPSRC Doctoral Training Partnership (DTP) and the partial support from the EPSRC Research Grant No: EP/N018737/1 on the "Quiet Aerofoils on the Next Generation" to fund the equipment.

\section{References}

[1] G. Corbetta, European Wind Energy Association, A. Ho, I. Pineda, "Wind energy scenarios for 2030", European Wind Energy Association, pp. 3-15, (2015).

[2] S. Oerlemans, M. Fisher, T. Maeder, K. Kögler, "Reduction of Wind Turbine Noise Using Optimized Airfoils and Trailing-Edge Serrations". AIAA Journal 47, 1470 (2009).

[3] A.Wolf, T.H. Lutz, W. Würz, E. Krämer, O. Stalnov and A. Seifert, "Trailing edge noise reduction of wind turbine blades by active flow control," Wind Energy; 18, 909 (2015).

[4] A.M. Knepper, "Examination of three candidate technologies for high-lift devices on an aircraft wing," Ph.D. thesis, Cranfield University, 2005.

[5] J. Hurault, A. Gupta, E. Sloth, N. Nielsen, A. Borgoltz, P. Ravetta, "Aeroacoustic wind tunnel experiment for serration design optimisation and its application to a wind turbine rotor", In $6^{\text {th }}$ International Meeting on Wind Turbine Noise, Glasgow (2015).

[6] T.P. Chong, A. Vathylakis, "On the aeroacoustic and flow structures developed on a flat plate with a serrated sawtooth trailing edge", J Sound Vib 354, 65 (2015).

[7] D.J. Moreau, C.J. Doolan, "Noise-Reduction Mechanism of a Flat-Plate Serrated Trailing Edge". AIAA Journal. Vol. 51, (2013), pp.2513-2522.

[8] M. Gruber, P.F. Joseph, T.P. Chong, "On the mechanisms of serrated airfoil trailing edge noise reduction", 17th AIAA/CEAS Aeroacoustics Conference Proceedings, AIAA Paper 2011-2781, pp. 5-8, (2011), Portland, Oregon.

[9] K. Braun, N. Van der Borg, A. Dassen, F. Doorenspleet, A. Gordner, J. Ocker and R. Parchen, "Serrated trailing edge noise (STENO)", in Proceedings of the European Wind Energy Conference, Nice, France, James \& James (Science Publishers) Ltd., London, UK., (1999).

[10] M.S. Howe, “Aerodynamic noise of a serrated trailing edge". J Fluids Struct 5, 33 (1990).

[11] O. Rodriguez, "Base drag reduction by control of the three-dimensional unsteady vortical structure", Exp Fluids 11, 218 (1991).

[12] A. Vathylakis, C.C. Paruchuri , T.P. Chong, P. Joseph, "Sensitivity of Aerofoil Self Noise Reductions to Serration Flap Angles", 22nd AIAA/CEAS Aeroacoustics Conference, AIAA Paper 2016-2837, (2016), Lyon, France.

[13] R. Amiet, "Noise due to turbulent flow past a trailing edge". J Sound Vib 47, 387 (1976).

[14] M. Gruber, P. Joseph, M. Azarpeyvand, "An experimental investigation of novel trailing edge geometries on airfoil trailing edge noise reduction", 19 $9^{\text {th }}$ AIAA/CEAS Aeroacoustics Conference, AIAA Paper 2013-2011, (2013), Berlin, Germany.

[15] B. Lyu, M. Azarpeyvand, S. Sinayoko, "A trailing-edge noise model for serrated edges", $21^{\text {st }}$ AIAA/CEAS Aeroacoustics Conference and Exhibit, AIAA Paper 2015-2362, (2015), Dallas, Texas.

[16] S. Probsting, "Coherent Structures at the Serrated Trailing-Edge of a NACA 0012", Master of Science Thesis, Delft University of Technology, 2011.

[17] C.A. León, D. Ragni, S. Pröbsting, F. Scarano, J. Madsen, "Flow topology and acoustic emissions of trailing edge serrations at incidence". Exp Fluids. (2016), 57:91, doi: 10.1007/s00348-016-2181-1.

[18] L. Jones, R. Sandberg, "Acoustic and hydrodynamic analysis of the flow around an aerofoil with trailing-edge serrations". Journal of Fluid Mech., 706, pp. 295-322, (2012).

[19] T. Dassen, R. Parchen, J. Bruggeman, F. Hagg, "Results of a wind tunnel study on the reduction of airfoil self-noise by the application of serrated blade trailing edges", Proceedings of the European Union Wind Energy Conference and Exhibition (Göteborg, Sweden), (1996).

[20] M.S. Howe, "Noise produced by a sawtooth trailing edge". The Journal of the Acoustical Society of America, 90, 482 (1991) 